\title{
Convulsion following intravenous fluorescein angiography
}

\author{
S P KELLY, ${ }^{1} \mathbf{N}$ J G MacDERMOTT, ${ }^{2}$ D C SAUNDERS, ${ }^{1}$ and F N LEACH ${ }^{3}$ \\ From the 'Manchester Royal Eye Hospital, the ${ }^{2}$ Department of Neurology, Manchester Royal Infirmary, \\ and the ${ }^{3}$ North Western Regional Drug Information Service
}

SUMmaRY Tonic-clonic seizures followed intravenous fluorescein injection for fundus angiography in a 47-year-old male. Despite precautions this adverse reaction recurred on re-exposure to intravenous fluorescein.

Fundus fluorescein angiography (FFA) is a well established technique in ophthalmic practice. Sodium fluorescein 5 to $10 \mathrm{ml}$ is given by intravenous injection in concentrations of 5 to $25 \%$. Mild adverse reactions are common; severe adverse reactions are exceptional.

We report a unique case of grand mal seizures following intravenous fluorescein.

\section{Case report}

A 47-year-old male with axial myopia and myopic chorioretinal degeneration developed reduction of left central vision. The visual acuity was $6 / 6$ Snellen right and 1/60 left. A fresh left macular haemorrhage was present. The patient was otherwise well.

He had been investigated six years previously for left sided paraesthesia, and neurological examination with cranial computed tomography gave normal results. Twenty years ago brief episodes of loss of consciousness had occurred, at which time investigations, including electroencephalography gave normal results. There was no history of anticonvulsant treatment.

Sodium fluorescein, $5 \mathrm{ml}$ of $25 \%$ solution, prepared at a local hospital pharmacy was injected intravenously for angiography after the pupils were dilated with $10 \%$ phenylephrine and $1 \%$ cyclopentolate eyedrops. No immediate adverse events occurred, but headache was experienced one hour later. Two hours after the fluorescein injection, while returning home, generalised tonic-clonic seizures with incontinence occurred. Prompt examination by his general practitioner gave normal results. A Correspondence to Simon Kelly, FRCSEd, Manchester Royal Eye Hospital, Oxford Road, Manchester M13 9WH. neurological consultation, cranial computed tomography, and digital subtraction intravenous angiography of the cerebral circulation also gave normal results. No adverse reaction to intravenous contrast medium injection occurred. The results of electroencephalography were normal apart from occasional $\theta$ activity in the right temporal region on hyperventilation. An untreatable subretinal neovascular membrane involving the left foveal avascular zone was demonstrated on the fluorescein angiogram.

Four months later similar events occurred in the right eye. The acuity was now $1 / 60$ right and left. Further fluorescein angiography was requested. Clobazam $10 \mathrm{mg}$ was given orally one hour prior to angiography, which was followed by bed rest under nursing observation. Headache occurred after the angiogram. Ninety minutes after the angiogram generalised tonic-clonic seizures occurred, with loss of consciousness. This brief episode resolved spontaneously. At present he remains otherwise well.

\section{Discussion}

Mild adverse reactions to intravenous fluorescein such as nausea and vomiting occur frequently. Severe adverse reactions which involve the respiratory, cardiac, or neurological systems are very unusual. The Fluorescein Angiography Complication Survey, a postal survey of adverse reactions to intravenous fluorescein, estimated tonic-clonic seizures to occur in 1:13900 angiograms; clinical details were not given.' Seizures following intrathecal sodium fluorescein have been reported. ${ }^{2}$ Topical fluorescein eyedrops have also caused generalised tonic-clonic seizures. ${ }^{3}$ While we are unaware of any other published reports of convulsions after FFA, one other 
case wherein this type of adverse reaction occurred has been reported to the Committee on Safety of Medicines (CSM, personal communication).

There is little doubt that the convulsion in our patient was precipitated by FFA as it happened within two hours of the injection and followed reexposure to a subsequent batch of fluorescein. This reaction appears to be an idiosyncratic reaction to the $25 \%$ sodium fluorescein injection. It is unlikely that the mydriatic eyedrops used were responsible, as they were used uneventfully on other occasions in this patient. As the convulsions occurred 1 1/2-2 hours after the injection, reaction to a metabolite is probable. Drug induced seizures are unusual, occurring in 1.3 per 1000 medical inpatients. ${ }^{4}$

Prophylaxis of this rare reaction is difficult. Premedication with a short acting anticonvulsant and subsequent bed rest failed to prevent a seizure from recurring. Fluorescein angiography in patients known to have had previous seizures should be assessed cautiously. Postangiogram observation may be prudent in such patients. Headache following fluorescein angiography may herald a convulsion.

\section{References}

1 Yannuzzi LA, Rohrer KT, Tindel LJ, et al. Fluorescein angiography complicaton survey (FACS). Ophthalmology 1986; 93: 611-7.

2 Mees K, Beyer A. Acute neurological complication following intrathecal fluorescein injection. Laryngol Rhinol Otol (Stuttg) 1982; 61: 102-4.

3 Cohn CC, Jocson VL. A unique case of grand mal seizures after Fluress. Ann Ophthalmol 1981; 13: 1379-80.

4 Jick H, Slone D, Shapiro S, et al. Boston Collaborative Drug Surveillance Program. Drug-induced convulsions. Lancet 1972; ii: 677-9.

Accepted for publication 24 November 1988. 